\title{
Comparação dos Stents Farmacológicos vs. Stents Convencionais para o Tratamento do Infarto Agudo do Miocárdio com Supradesnivelamento do Segmento ST: Resultados do Registro EINSTEIN
}

\author{
Marco Aurélio de Magalhães ${ }^{1}$, Fábio Sandoli de Brito Jr. ${ }^{1}$, Breno Oliveira Almeida ${ }^{1}$, Alexandre Abizaid ${ }^{1}$, \\ Ivanise Gomes ${ }^{1}$, Teresa Cristina Nascimento ${ }^{1}$, Marco Antonio Perin ${ }^{1}$
}

\section{RESUMO}

Fundamentos: Os estudos randomizados que testaram os stents farmacológicos no infarto agudo do miocárdio com supradesnivelamento do segmento ST (IAMSST) demonstraram resultados conflitantes. Além disso, a trombose do stent e o seguimento tardio pouco reportado permanecem como os grandes limitantes à expansão da aplicação desses dispositivos no IAMSST. Objetivo: Comparar as taxas de eventos cardíacos adversos maiores combinados (ECAM) e as taxas de trombose do stent em pacientes não-selecionados tratados com stents convencionais ou farmacológicos no IAMSST e alocados de forma não-randômica. Método: Entre janeiro de 2001 e julho de 2007, 251 pacientes consecutivos com IAMSST foram submetidos a angioplastia primária ou de resgate com implante de stent convencional $(n=112)$ ou farmacológico $(n=139)$, no Hospital Israelita Albert Einstein - São Paulo, SP. Investigamos a incidência de ECAM (óbito, infarto do miocárdio e revascularização do vaso-alvo [RVA]) e as taxas de RVA e trombose do stent. Resultados: No total, a média das idades foi de $63 \pm 13$ anos, sendo $77 \%$ dos pacientes do sexo masculino. No seguimento mediano de 2,8 anos $(1,3-4,4)$, a incidência de ECAM foi maior nos pacientes tratados com stents convencionais, em comparação com os tratados com stents farmacológicos $(25,2 \%$ vs. $10,1 \%$; p $<0,01)$, fundamentalmente pela maior mortalidade tanto global $(17,8 \%$ vs. $5,1 \% ; p<0,01)$ como cardíaca $(15,9$ vs. 4,$3 ; p<0,01)$ no grupo dos stents convencionais. A taxa de RVA foi de $7,5 \%$ no grupo dos stents convencionais e de $3,6 \%$ no grupo dos stents farmacológicos $(p=0,25)$. Não observamos diferenças significativas na incidência de trombose do stent $(3,7 \%$ vs. $2,2 \% ; p=0,7)$. Conclusões: Nessa série observacional, o uso dos stents farmacológicos no IAMSST apresentou menor taxa de ECAM em comparação aos stents convencionais no seguimento clínico tardio.

DESCRITORES: Infarto do miocárdio. Contenedores. Stents farmacológicos. Angioplastia transluminal percutânea coronária. Avaliação de resultados.
1 Hospital Israelita Albert Einstein - São Paulo, SP.

Correspondência: Marco Aurélio de Magalhães. Rua Xavier da Veiga, 81 - apto. 194 - Santana - São Paulo, SP - CEP 02021-040

E-mail mademagalhaes@yahoo.com.br

Recebido em: 25/5/2008 • Aceito em: 1\%/8/2008

\section{SUMMARY}

Long-Term Outcomes of Drug-eluting Stents Compared to Bare-metal Stents for Acute ST-

Elevation Myocardial Infarction:

Results from the EINSTEIN Registry

Background: Randomized clinical trials comparing drugeluting stents (DES) with bare metal stents (BMS) in the setting of ST-segment elevation myocardial infarction (STEMI) have been showing conflicting results. Additionally, issues regarding stent thrombosis and the lack of long-term clinical outcomes have limited the widespread use of DES in STEMI. Objective: To compare the rates of major adverse cardiac events (MACE) and stent thrombosis in unselected patients who underwent primary or rescue percutaneous coronary intervention with BMS implantation with those treated with DES. Methods: Between January 2001 and July 2007, 251 consecutive patients with STEMI underwent $\mathrm{PCl}$ with BMS $(\mathrm{n}=112)$ or DES $(\mathrm{n}=139)$. We investigated the incidence of MACE, including death, myocardial infarction and target vessel revascularization (TVR), as well as stent thrombosis rates. Results: Overall, the mean age was $63 \pm 13$ years-old and $77 \%$ were male. The median clinical follow-up was 2.8 years (1.3-4.4). The cumulative incidence of MACE was higher in patients who received BMS as compared with DES $(25.2 \%$ vs. $10.1 \%$; p < 0.01$)$ driven by a higher incidence of all-cause mortality $(17.8 \%$ vs. $5.1 \%$; p < 0.01), mainly cardiac death (15.9 vs. 4.3; $p<0.01)$. TVR was detected in 8 patients $(7.5 \%)$ with BMS and in 5 patients $(3.6 \%)$ with DES $(p=0.25)$. The incidence of stent thrombosis was similar between groups (3.7\% vs. $2.2 \% ; p=0.7)$. Conclusions: In this observational series, the use of DES in the setting of STEMI showed lower rates of MACE compared to BMS at the long-term follow-up.

DESCRIPTORS: Myocardial infarction. Stents. Drug-eluting stents. Angioplasty, transluminal, percutaneous coronary. Outcome assessment. 
A intervenção coronária primária é a estratégia de escolha para o tratamento do infarto agudo do miocárdio com supradesnivelamento do segmento ST (IAMSST), quando realizada precocemente por operadores experientes ${ }^{1,2}$. O implante de stent na angioplastia primária ou de resgate está associado a menor taxa de revascularização do vaso-alvo (RVA) ${ }^{3,4}$ e, possivelmente, a mortalidade em subgrupos selecionados em comparação com a angioplastia convencional com balão ${ }^{5,6}$. A primeira geração de stents farmacológicos, que vem sendo recentemente testada no IAMSST, tem apresentado resultados conflitantes quanto a sua eficácia, em comparação com os stents convencionais ${ }^{7,8}$. Além disso, questionamentos acerca do incremento tardio das taxas de trombose e a pouca disponibilidade de dados referentes ao seguimento tardio desses pacientes constituem os grandes limitantes à expansão do uso desses dispositivos no universo da prática clínica do tratamento do IAMSST.

Dessa forma, avaliamos os resultados muito tardios de pacientes não-selecionados com IAMSST tratados com stents farmacológicos em comparação com aqueles submetidos a implante de stents convencionais num único centro.

\section{MÉTODO}

\section{População}

No período compreendido entre janeiro de 2001 e julho de 2007, no Hospital Israelita Albert Einstein, São Paulo, SP, foram admitidos 251 pacientes consecutivos com IAMSST tratados por intervenção coronária primária ou de resgate e implante de stent na artéria relacionada ao evento. Destes, 139 receberam ao menos um stent farmacológico e fazem parte do Registro EINSTEIN, que reúne todos os pacientes tratados com esses dispositivos desde 16 de maio 2002. Foram comparados com 112 pacientes tratados com stents convencionais, no período imediatamente anterior à disponibilidade dos stents farmacológicos ou posteriormente, por decisão do operador, em geral quando o diâmetro do vaso tratado era $\geq 4 \mathrm{~mm}$. Comparamos, retrospectivamente, a taxa de eventos cardíacos adversos maiores combinados (ECAM) nos dois grupos. Os critérios de exclusão deste estudo foram a angioplastia convencional e os pacientes admitidos com sintomas há mais de 24 horas.

\section{Definições}

Considerou-se infarto agudo do miocárdio a presença de dor torácica prolongada, associada a supradesnivelamento do segmento ST $>1 \mathrm{~mm}$ em pelo menos duas derivações contíguas ou bloqueio de ramo esquerdo agudo ou presumivelmente novo. Os pacientes foram estratificados na sua admissão e evolução conforme a classificação de Killip. Definiu-se choque cardiogênico (Killip IV) como hipotensão persistente (pressão arterial sistólica $<90 \mathrm{mmHg}$ por no mínimo trinta minutos) ou necessidade de se utilizar drogas vasoativas. Doença coronária multiarterial foi definida como a presença de pelos menos dois vasos epicárdicos com estenose superior a 50\% por estimativa visual.

Os ECAM incluíram óbito cardíaco, reinfarto e RVA, agrupados de forma hierárquica. O óbito foi classificado como não-cardíaco somente após comprovação inequívoca da causa, enquanto a óbito cardíaco foram imputados os casos definidos bem como os de causa desconhecida. O reinfarto foi definido como a presença de novos sintomas anginosos acompanhados por elevação enzimática, sendo também considerados reinfarto os casos comprovadamente não relacionados ao vaso-alvo. RVA foi definida como qualquer reintervenção no vaso-alvo por reestenose ou trombose, tanto cirúrgica como percutânea. Revascularização da lesão-alvo (RLA) foi definida como reintervenção no seguimento tratado, incluindo os $5 \mathrm{~mm}$ proximais e distais ao implante do stent.

A trombose do stent foi classificada de acordo com os critérios adotados pelo Academic Research Consortium $^{9}$, que classifica a trombose de stents como definitiva, provável ou possível e, de acordo com o intervalo de tempo, como precoce (0 a 30 dias), tardia (31 a 365 dias) e muito tardia (> 365 dias).

\section{Desfechos clínicos primários e secundários}

O desfecho primário do estudo foi a comparação da taxa de ECAM no seguimento clínico entre os pacientes tratados com stents convencionais e aqueles tratados com stents farmacológicos. Como desfechos secundários, compararam-se as taxas de RVA e de trombose do stent. Além disso, avaliamos o tempo de distribuição de todos os eventos individuais e combinados, de acordo com sua ocorrência, em intra-hospitalar até trinta dias, primeiro, segundo, terceiro, quarto e quinto anos de seguimento clínico. Todos os eventos foram adjudicados por dois cardiologistas intervencionistas.

\section{Intervenção coronária primária}

Os procedimentos foram realizados de acordo com as recomendações vigentes formalizadas nas diretrizes de intervenção coronária primária. Os procedimentos foram realizados exclusivamente pela via femoral e todos os pacientes receberam $300 \mathrm{mg}$ de clopidogrel antes ou imediatamente após o procedimento. Após o diagnóstico, administrou-se heparina não-fracionada na dose de $100 \mathrm{U} / \mathrm{kg}$ ou $70 \mathrm{U} / \mathrm{kg}$ no caso de uso concomitante de abciximab (ReoPro, Eli Lilly - Indianápolis, Indiana, Estados Unidos; Centocor - Horsham, Pensilvânia, Estados Unidos). O nível de anticoagulação foi monitorado utilizando-se o tempo de coagulação ativada (TCA) medido pelo equipamento Hemocron 801. Doses adicionais de heparina foram administradas quando necessário, objetivando-se atingir TCA 
Magalhães MA, et al. Comparação dos Stents Farmacológicos vs. Stents Convencionais para o Tratamento do Infarto Agudo do Miocárdio com Supradesnivelamento do Segmento ST: Resultados do Registro EINSTEIN. Rev Bras Cardiol Invas. 2008;16(3):279-288.

entre $250 \mathrm{~s}$ e $350 \mathrm{~s}$ ou entre $200 \mathrm{~s}$ e $250 \mathrm{~s}$ quando se fez uso concomitante de abciximab.

A escolha do stent ocorreu de acordo com o período, sendo utilizados exclusivamente stents convencionais de 5 de janeiro de 2001 a 10 de julho de 2002, quando houve o primeiro implante de stent farmacológico nesse contexto. A partir dessa data, a opção por stent convencional ou farmacológico ocorreu a critério do operador, exceto nos casos de vasos de grande calibre (> 4,0 mm), em que a preferência se manteve pelos stents convencionais. Deve-se mencionar também que a escolha entre os stents farmacológicos foi influenciada pela disponibilidade comercial desses stentes na instituição (Cypher, 16 de maio de 2002; Taxus, 2 de maio de 2003; e CoStar, 27 de abril de 2006).

O uso adjuvante de dispositivos de trombectomia também ocorreu de acordo com a opção do operador, sendo utilizados o cateter Pronto V3 (Vascular Solutions - Minneapolis, Minnesota, Estados Unidos) ou X-sizer (EV3 - Plymouth, Minnesota, Estados Unidos). O tempo de manutenção do clopidogrel foi definido pelo cardiologista de referência.

\section{Análise angiográfica quantitativa}

Realizou-se análise angiográfica quantitativa com o sistema validado de detecção de bordas CASS II (Pie Medical - Maastricht, Holanda $)^{10}$. Nos casos em que a artéria relacionada ao infarto se apresentava ocluída, foram utilizadas as primeiras projeções após o restabelecimento do fluxo coronário anterógrado, conseguido pela passagem do fio-guia 0,014" ou após a pré-dilatação, para medir o comprimento da lesão. Definiu-se como sucesso angiográfico a presença de estenose angiográfica residual menor que $30 \%$ e fluxo anterógrado normal (TIMI 3).

\section{Seguimento clínico}

O seguimento clínico foi obtido de forma retrospectiva por meio de contato telefônico ou eletrônico (e-mail) com o próprio paciente, familiares ou com o médico de referência e pela consulta de arquivos hospitalares. Obteve-se informação quanto ao seguimento clínico em 97,6\% dos pacientes. Não obtivemos informações quanto ao seguimento clínico em 5 (4,4\%) pacientes do grupo dos stents convencionais e em apenas $1(0,7 \%)$ paciente do grupo dos stents farmacológicos.

\section{Análise estatística}

As variáveis contínuas são representadas pela sua média \pm 1 desvio padrão, enquanto as variáveis categóricas são representadas pela freqüência absoluta ou porcentual. A comparação das variáveis categóricas entre os grupos foi realizada pelo teste do qui-quadrado ou exato de Fisher. As variáveis contínuas foram comparadas com teste $t$ de Student. As estimativas da sobrevida livre de eventos foram geradas pelo método produto-limite de Kaplan-Meier e as diferenças entre os grupos, testadas por log-rank. Todos os resultados reportados são bicaudais e considerou-se $p<0,05$ indicativo de significância estatística. Utilizou-se o software SPSS 13.0 Inc. (Chicago, Illinois, Estados Unidos).

\section{RESULTADOS}

No total, a média aritmética das idades foi de $63 \pm 13$ anos, sendo $77 \%$ homens e 22,7\% diabéticos. As características basais e clínicas, expostas na Tabela 1, foram semelhantes entre os grupos, com exceção das pressões sistólica $(123 \pm 35 \mathrm{mmHg}$ vs. $133 \pm 28 \mathrm{mmHg} ; \mathrm{p}=0,03)$ e diastólica $(77 \pm 24 \mathrm{mmHg}$ vs. $84 \pm 19 \mathrm{mmHg} ; p=0,03)$ na admissão, significantemente inferiores no grupo do stent convencional.

Dentre as características angiográficas e do procedimento, representadas na Tabela 2, 58,8\% dos vasos relacionados ao infarto apresentavam fluxo TIMI 0, 2,4\% das intervenções envolveram enxertos venosos e $43 \%$ envolveram a artéria descendente anterior. A taxa de sucesso global foi de $90 \%$. O abciximab adjunto foi utilizado em $64,5 \%$ dos casos. Entre os pacientes que receberam stent farmacológico, 55\% receberam stent eluidor de sirolimus, $37 \%$ receberam stent eluidor de paclitaxel na plataforma do stent Taxus e $8 \%$, do stent CoStar. Destacamos a presença do maior diâmetro dos stents convencionais implantados em comparação com os stents farmacológicos $(3,4 \pm 0,5 \mathrm{~mm}$ vs. 3,1 $\pm 0,3 \mathrm{~mm}$; $\mathrm{p}<0,01)$. Por outro lado, os stents farmacológicos foram implantados com maior pressão de liberação $(14,7 \pm 2,4$ atm vs. 16,9 $\pm 2,9$ atm; $p<0,01)$ e mais freqüentemente pós-dilatados $(19,0 \%$ vs. $32,8 \%$; p = 0,02). O comprimento total dos stents bem como o uso de dispositivos de trombectomia adjunto não diferiu significativamente entre os grupos. O tamanho do infarto, estimado pelo pico da enzima CK-MB, foi semelhante entre os grupos $(206 \pm 178 \mathrm{Ul} / \mathrm{ml}$ vs. $203 \pm 170 \mathrm{Ul} / \mathrm{ml} ; \mathrm{p}=0,92)$.

O tempo de manutenção da dupla antiagregação plaquetária no grupo dos stents farmacológicos foi de $1,3 \pm 0,8$ ano.

\section{Desfechos clínicos primário e secundário: resultados cumulativos}

No seguimento clínico tardio (seguimento mediano de 2,8 anos [1,3-4,4]; amplitude de 0 a 6,6 anos), detectamos 41 ECAM $(16,7 \%)$ nessa população (Tabela 3). Observamos maior incidência de ECAM nos pacientes submetidos a implante de stents convencionais em comparação com os pacientes tratados com stents farmacológicos (25,2\% vs. 10,1\%; p < 0,01), em decorrência, fundamentalmente, de redução da incidência de óbito cardíaco $(15,9 \%$ vs. $4,3 \%$; $p<0,01)$. Na população global, documentamos, de forma conclusiva, como óbito não-cardíaco apenas 3 casos: 2 casos na coorte de stent convencional (1 paciente com neoplasia de reto e 1 paciente com neoplasia de bexiga), enquanto na coorte dos stents farmacológicos 
TABELA 1

Características demográficas e clínicas basais dos pacientes

\begin{tabular}{lcccc}
\hline Variáveis & $\begin{array}{c}\text { Total } \\
\mathbf{n = 2 5 1}\end{array}$ & $\begin{array}{c}\text { Stent convencional } \\
\mathbf{n = 1 1 2}\end{array}$ & $\begin{array}{c}\text { Stent farmacológico } \\
\mathbf{n = 1 3 9}\end{array}$ & $\begin{array}{c}\text { Valor } \\
\mathbf{d e ~} \mathbf{p}\end{array}$ \\
\hline Homens, \% & 77 & 75,9 & 78,4 & 0,65 \\
Idade, anos & $63 \pm 13$ & $63,5 \pm 15$ & $62,9 \pm 12$ & 0,69 \\
Hipertensão arterial sistêmica, \% & 57,8 & 55,4 & 59,4 & 0,58 \\
Diabetes melito, \% & 22,7 & 21,7 & 23,3 & 0,52 \\
DMNID & 18,7 & 16,3 & 20,3 & 0,3 \\
DMID & 4 & 5,4 & 37,9 & 1 \\
Tabagista atual, \% & 34,4 & 29,3 & 31,9 & 0,78 \\
Hipercolesterolemia, \% & 31,8 & 31,8 & 38,9 & 0,03 \\
Antecedente familiar para DAC, \% & 38,1 & 37 & $133 \pm 28$ & 0,03 \\
PAS na admissão, mm Hg & $129 \pm 32$ & $123 \pm 35$ & $80 \pm 19$ & 18 \\
PAD na admissão, mm Hg & $81 \pm 21$ & $77 \pm 24$ & 15 & 0,09 \\
Freqüência cardíaca na admissão, b/min & $78 \pm 20$ & $75 \pm 22$ & $7(5,0)$ & 0,36 \\
Killip III/IV, \% & 17 & 20 & $50,7 \pm 10$ & 0,76 \\
Angioplastia de resgate, \% & $11(4,4)$ & $4(3,6)$ & $203 \pm 170$ \\
Fração de ejeção doVE, \% & $50 \pm 11$ & $50,0 \pm 12$ & 0,66 \\
Pico de CK-MB, Ul/ml & $204 \pm 171$ & $206 \pm 178$ & 0,92 \\
\hline
\end{tabular}

$\mathrm{DAC}=$ doença arterial coronária; DMID = diabetes melito insulino-dependente; DMNID = diabetes melito não-insulino-dependente; $\mathrm{n}=$ número de pacientes; PAD = pressão arterial diastólica; PAS = pressão arterial sistólica; VE = ventrículo esquerdo.

documentamos apenas 1 óbito não-cardíaco (1 paciente com traumatismo cranioencefálico com marcadores enzimáticos normais e eletrocardiograma sem novas ondas Q patológicas). Observamos tendência de menor taxa de revascularização por reestenose clínica nos pacientes tratados com stents farmacológicos em comparação com os stents convencionais, embora sem atingir significância estatística $(6,5 \%$ vs. $2,9 \% ; p=0,18)$. As curvas das estimativas da sobrevida livre de ECAM e de revascularização do vaso-alvo estão representadas nas Figuras 1 e 2, respectivamente.

\section{Trombose dos stents}

As taxas de trombose relacionadas ao stent, avaliadas pelo critério amplo ou restringindo-se aos quadros definitivos, não foram estatisticamente diferentes entre os stents convencionais e farmacológicos (4 [3,7\%] vs. $3[2,2 \%], p=0,70 ; 1[0,9 \%]$ vs. $2[1,4 \%], p=1,0)$. Todos os 7 (2,9\%) eventos trombóticos foram adjudicados e analisados detalhadamente quanto às características clínicas e angiográficas do procedimento índice, tempo de ocorrência, manifestação clínica e terapia antiplaquetária vigente no momento do evento, e encontram-se resumidos na Tabela 4. Observa-se que somente 2 pacientes estavam em regime de dupla antiagregação no momento do evento.

\section{Eventos após três anos de seguimento}

Conforme exposto na Tabela 5, observamos a quase totalidade dos eventos combinados (78\%) no primeiro e no segundo anos de seguimento clínico. Após o terceiro ano de seguimento, nota-se significativa redução da taxa de eventos em ambos os grupos de stents.

\section{DISCUSSÃO}

Os principais achados deste estudo são: 1) segundo nosso conhecimento, esta foi a primeira vez em que foi reportado, na literatura, o seguimento muito tardio (após três anos) de pacientes não-selecionados e tratados com stents farmacológicos na fase aguda do IAMSST; 2) menor taxa de ECAM combinados observada no grupo de pacientes tratados com stents farmacológicos; e 3) ausência de fenômenos trombóticos além do primeiro ano (muito tardios) nos pacientes tratados com stents farmacológicos.

As taxas de reestenose e de RVA no contexto da angioplastia primária provenientes de estudos randomizados situam-se em cerca de $10 \%$ em doze meses ${ }^{3}$. Cabe ressaltar que a maioria desses estudos apresentou critérios rígidos de inclusão, dificultando a transposição desses resultados para a prática clínica diária, em que as taxas de reestenose no infarto podem superar 30\% nos casos de reestudo angiográfico sistemático de pacientes não-selecionados ${ }^{11}$. Esse aspecto é relevante, pois, além da necessidade de nova intervenção, a reestenose intra-stent se manifesta freqüentemente como síndrome coronária aguda e infarto ${ }^{12,13}$, o que determina grande impacto na morbidade e possivelmente na mortalidade ${ }^{14}$. Assim, também no IAMSST 
TABELA 2

Características angiográficas e do procedimento

\begin{tabular}{|c|c|c|c|c|}
\hline Variáveis & $\begin{array}{c}\text { Total } \\
\mathrm{n}=251\end{array}$ & $\begin{array}{l}\text { Stent convencional } \\
\qquad n=112\end{array}$ & $\begin{array}{l}\text { Stent farmacológico } \\
\qquad n=139\end{array}$ & $\begin{array}{l}\text { Valor de } \\
\quad p\end{array}$ \\
\hline \multicolumn{5}{|l|}{ Artéria relacionada, \% } \\
\hline TCE & 0,8 & 0,9 & 0,7 & \\
\hline DA & 43,0 & 35,7 & 48,9 & \\
\hline$C D$ & 39,4 & 46,4 & 33,8 & 0,18 \\
\hline CX & 12,4 & 13,4 & 11,5 & \\
\hline PVS & 2,4 & 0,9 & 3,6 & \\
\hline \multicolumn{5}{|l|}{ Fluxo TIMI pré-procdimento, \% } \\
\hline Grau 0 & 58,8 & 66,4 & 52,3 & \\
\hline Grau 1 & 10,4 & 10,9 & 10 & 0,09 \\
\hline Grau 2 & 10,4 & 8,2 & 12,3 & \\
\hline Grau 3 & 20,4 & 14,5 & 25,4 & \\
\hline \multicolumn{5}{|l|}{ Fluxo TIMI pós-procedimento, \% } \\
\hline Grau 1 & 1,3 & 1,8 & 0,8 & \\
\hline Grau 2 & 8,3 & 10,9 & 6,2 & 0,3 \\
\hline Grau 3 & 90,4 & 87,3 & 93,1 & \\
\hline Abciximab adjunto, \% & 64,5 & 62,5 & 66,2 & 0,47 \\
\hline Trombectomia, \% & 6,4 & 9,8 & 3,6 & 0,12 \\
\hline Comprimento total do stent, $(\mathrm{mm})$ & $26 \pm 14$ & $25,1 \pm 14$ & $27,2 \pm 14$ & 0,25 \\
\hline Diâmetro stent, (mm) & $3,2 \pm 0,5$ & $3,4 \pm 0,5$ & $3,1 \pm 0,3$ & $<0,01$ \\
\hline Multiarterial, \% & 67,6 & 64,9 & 69,9 & 0,41 \\
\hline Stent/paciente & $1,27 \pm 0,5$ & $1,21 \pm 0,4$ & $1,24 \pm 0,6$ & 0,58 \\
\hline Bifurcação, \% & 7,2 & 6,3 & 7,2 & 0,8 \\
\hline \multicolumn{5}{|l|}{ Diâmetro de referência do vaso } \\
\hline \multicolumn{5}{|l|}{ Diâmetro de referência do vaso } \\
\hline \multicolumn{5}{|l|}{ Diâmetro luminal mínimo } \\
\hline $\begin{array}{l}\text { pré-procedimento, mm } \\
\text { Diâmetro luminal mínimo }\end{array}$ & \multicolumn{3}{|c|}{ Diâmetro luminal mínimo } & $<0,01$ \\
\hline pós-procedimento, mm & \multicolumn{3}{|c|}{ Diâmetro de estenose } & 0,52 \\
\hline \multicolumn{2}{|l|}{ Diâmetro de estenose } & $91 \pm 14$ & $86 \pm 19$ & $<0,01$ \\
\hline pós-procedimento, \% & $18 \pm 9$ & $18,7 \pm 8$ & $18,2 \pm 10$ & 0,69 \\
\hline Comprimento da lesão, mm & $14,9 \pm 7,5$ & $14,7 \pm 7,0$ & $15,0 \pm 7,8$ & 0,76 \\
\hline Stent direto, \% & 34,5 & 28,6 & 39,4 & 0,09 \\
\hline Pós-dilatação, \% & 26,5 & 19 & 32,8 & 0,02 \\
\hline Pressão de liberação, atm & $16,0 \pm 2,9$ & $14,7 \pm 2,4$ & $16,9 \pm 2,9$ & $<0,01$ \\
\hline
\end{tabular}

$\mathrm{CD}=$ artéria coronária direita; $\mathrm{CX}=$ artéria circunflexa; $\mathrm{DA}=$ artéria descendente anterior; $\mathrm{n}=$ número de pacientes; $\mathrm{PVS}=$ ponte de veia safena; TCE = tronco de coronária esquerda.

encontra-se uma lacuna oportuna para a aplicação dos stents farmacológicos, objetivando-se a redução das taxas de reestenose. Os estudos randomizados que testaram o stent eluidor de sirolimus em comparação com os stents convencionais atingiram significância estatística com relação a seus desfechos primários pré-especificados: $\mathrm{TYPHOON}^{8}$, falência do vaso-alvo aos doze meses (7,3\% vs. 14,3\%; $p=0,004)$; SESAMI ${ }^{15}$, reestenose binária aos doze meses (9,3\% vs. 21,3\%; $\mathrm{p}=0,03)$; STRATEGY ${ }^{16}$, eventos adversos combinados ou reestenose binária aos oito meses (19\% vs. 50\%; $\mathrm{p}<0,01)$; e MULTISTRATEGY ${ }^{17}$, ECAM aos oito meses 
TABELA 3

Resultados cumulativos

\begin{tabular}{lcccc}
\hline Desfecho & $\begin{array}{c}\text { Total } \\
\mathbf{n = 2 5 1}\end{array}$ & $\begin{array}{c}\text { Stent convencional } \\
\mathbf{n}=\mathbf{1 1 2}\end{array}$ & $\begin{array}{c}\text { Stent farmacológico } \\
\mathbf{n = 1 3 9}\end{array}$ & Valor de $\mathbf{p}$ \\
\hline Óbito global (\%) & $26(10,6)$ & $19(17,8)$ & $7(5,1)$ & $<0,01$ \\
Óbito cardíaco (\%) & $23(9,4)$ & $17(15,9)$ & $6(4,3)$ & $<0,01$ \\
Óbito não-cardíaco (\%) & $3(1,2)$ & $2(1,9)$ & $1(0,7)$ & 0,58 \\
Infarto do miocárdio (\%) & $15(6,1)$ & $8(7,5)$ & $7(5,1)$ & 0,59 \\
RVA (\%) & $13(5,3)$ & $8(7,5)$ & $5(3,6)$ & 0,25 \\
RLA (\%) & $11(4,5)$ & $7(6,5)$ & $4(2,9)$ & 0,18 \\
Trombose, critério amplo (\%) & $7(2,9)$ & $4(3,7)$ & $3(2,2)$ & 0,7 \\
Trombose definitiva (\%) & $3(1,2)$ & $1(0,9)$ & $2(1,4)$ & 1 \\
ECAM (\%) (hierárquico) & $41(16,7)$ & $27(25,2)$ & $14(10,1)$ & $<0,01$ \\
\hline
\end{tabular}

ECAM = eventos cardíacos adversos maiores; $\mathrm{n}$ = número de pacientes; RLA = revascularização da lesão-alvo; RVA = revascularização do vaso-alvo.

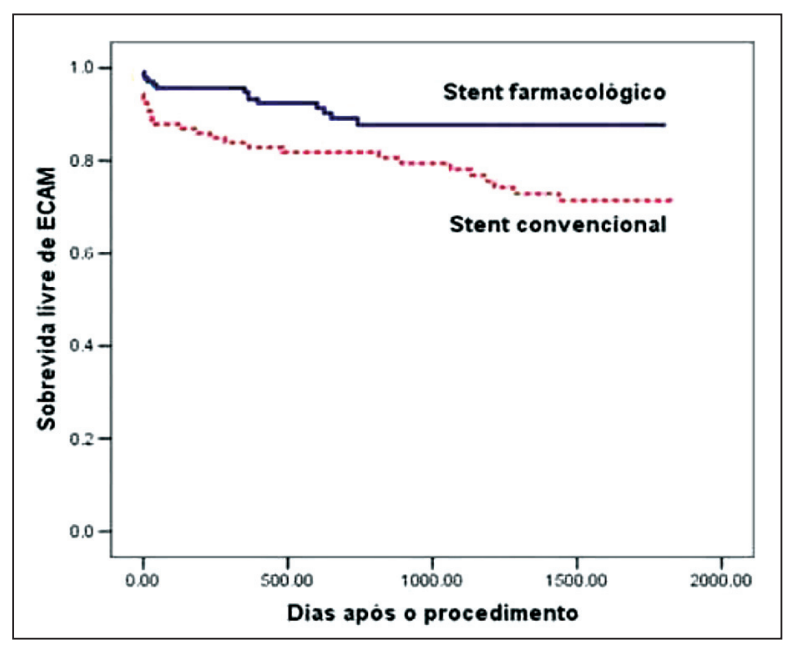

Figura 1 - Curvas de Kaplan-Meier da sobrevida livre de eventos cardíacos adversos maiores. Log-rank $\mathrm{p}=0,015$. ECAM $=$ eventos cardíacos adversos maiores.

(7,8\% vs. $14,5 \% ; p=0,004)$. Por outro lado, o estudo randomizado envolvendo stent com paclitaxel em comparação com o stent convencional não demonstrou diferença estatisticamente significante na taxa de eventos combinados aos doze meses $(8,8 \%$ vs. $12,8 \%$; $\mathrm{p}=0,09)^{7}$. Entretanto, é importante ressaltar que, ao contrário dos estudos anteriores, esse estudo não apresentou reestudo angiográfico sistemático, o que pode ter influenciado, em parte, os resultados em decorrência do conhecido fenômeno óculo-estenótico. Em nosso estudo, também baseado em seguimento clínico, observamos baixas taxas de reestenose clínica em ambos os grupos, no total em 5,3\% dos pacientes, e de acordo com o total global do estudo PASSION, de aproximadamente $6 \%{ }^{7}$, o que demonstra que as taxas de reestenose clínica no infarto são afetadas de

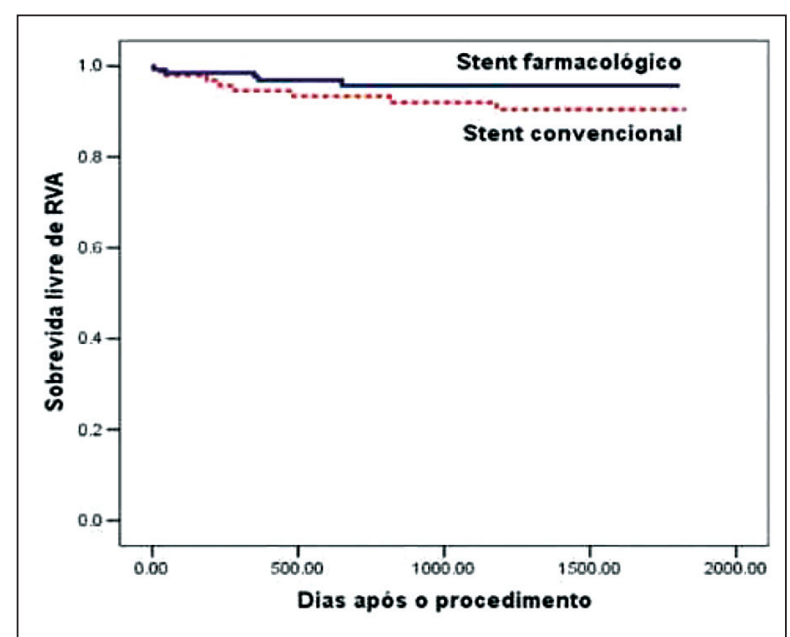

Figura 2 - Curvas de Kaplan-Meier da sobrevida livre de revascularização do vaso-alvo. Log-rank $\mathrm{p}=0,22$. RVA $=$ revascularização do vaso-alvo.

maneira conspícua pelo reestudo sistemático. Não obstante, observamos, nesses estudos, que as taxas de nova intervenção no vaso-alvo no IAMSST tratados com stents farmacológicos se situam ao redor de $5 \%{ }^{18}$, o que significa que houve redução expressiva em comparação com os estudos clássicos envolvendo stents convencionais ${ }^{3}$. Essa melhora indica que o mecanismo de ação dos stents farmacológicos está presente de forma inconteste, mesmo no IAMSST, e de fato recente estudo comprova que a perda tardia aos nove meses do stent eluidor de sirolimus foi de $0,12 \pm 0,43 \mathrm{~mm}$, em comparação com a perda tardia de 0,68 $\pm 0,57 \mathrm{~mm}$ no grupo dos stents convencionais $(p<0,001)^{19}$. Ao analisarmos os eventos individuais no presente estudo, porém, observamos que a redução dos eventos combinados em favor dos 
TABELA 4

Eventos trombóticos adjudicados nos stents convencionais e farmacológicos

\begin{tabular}{|c|c|c|c|c|c|}
\hline $\begin{array}{l}\text { Descrição do } \\
\text { procedimento índice }\end{array}$ & $\begin{array}{c}\text { Procedimento e } \\
\text { análise } \\
\text { angiográfica } \\
\text { quantitativa }\end{array}$ & $\begin{array}{c}\text { Tempo de } \\
\text { ocorrência }\end{array}$ & $\begin{array}{l}\text { Manifestação } \\
\text { clínica }\end{array}$ & $\begin{array}{c}\text { Terapia } \\
\text { antiplaquetária }\end{array}$ & $\begin{array}{l}\text { Definição } \\
\text { por } \\
\text { critério } \\
\text { ARC }\end{array}$ \\
\hline $\begin{array}{l}\text { \# Pac, } 89 \text { anos, } \\
\text { feminino } \\
\text { Julho } 2002 \text { - IAM } \\
\text { anterior - ICP da DA } \\
\text { com Bx. Sonic } \\
3,0 \times 18 \mathrm{~mm} \\
\text { FEVE: } 35 \%\end{array}$ & $\begin{array}{l}\text { DRV: 2,62 mm } \\
\text { (-) Abciximab } \\
\text { TCA: } 218 \mathrm{~s} \\
\text { (-) Pós-dilatação } \\
\text { Fluxo TIMI } 3 \text { pós }\end{array}$ & $\begin{array}{l}12 \text { horas } \\
\text { após ICP }\end{array}$ & PCR refratária & Dupla & Provável \\
\hline $\begin{array}{l}\text { \# Pac, } 39 \text { anos, } \\
\text { masculino } \\
\text { Dezembro } 2001 \text { - IAM } \\
\text { inferior - ICP da CD com } \\
\text { Bx. Sonic } 3,5 \times 13 \text { mm } \\
\text { FEVE: } 68 \%\end{array}$ & $\begin{array}{l}\text { DRV: } 3,12 \mathrm{~mm} \\
\text { (+) Abciximab } \\
\text { TCA: (-) Pós-dilatação } \\
\text { Fluxo TIMI } 3 \text { pós }\end{array}$ & 1.180 dias & $\begin{array}{c}\text { IAM inferior } \\
\text { com elevação } \\
\text { do segmento } \\
\text { ST }\end{array}$ & Aspirina & Definitiva \\
\hline $\begin{array}{l}\text { \# Pac, } 69 \text { anos, } \\
\text { masculino } \\
\text { Novembro } 2002 \text { - IAM } \\
\text { inferior - ICP da CD com } \\
\text { Express } 4,0 \times 28 \text { mm } \\
\text { FEVE: } 52 \%\end{array}$ & $\begin{array}{l}\text { DRV: 4,09 mm } \\
\text { (+) Abciximab } \\
\text { TCA: } 247 \text { s } \\
\text { (-) Pós-dilatação } \\
\text { Fluxo TIMI } 3 \text { pós }\end{array}$ & 1.062 dias & Morte súbita & Aspirina & Possível \\
\hline $\begin{array}{l}\text { \# Pac, } 79 \text { anos, } \\
\text { feminino } \\
\text { Setembro - } 2002 \text { IAM } \\
\text { inferior - ICP da CD com } \\
\text { Express } 3,0 \times 28 \text { mm } \\
\text { FEVE: } 65 \%\end{array}$ & $\begin{array}{c}\text { DRV: 2,84 mm } \\
\text { (-) Abciximab } \\
\text { TCA: - } \\
\text { (-) Pós-dilatação } \\
\text { Fluxo TIMI } 3 \text { pós }\end{array}$ & 892 dias & Morte súbita & Aspirina & Possível \\
\hline $\begin{array}{l}\text { \# Pac, } 63 \text { anos, } \\
\text { feminino } \\
\text { Fevereiro } 2004 \text { - IAM } \\
\text { inferior - ICP da CD com } \\
\text { Cypher } 3,0 \times 23 \mathrm{~mm} \text { e } \\
\text { Taxus } 3,0 \times 24 \mathrm{~mm} \\
\text { (overlapping) } \\
\text { FEVE: } 73 \%\end{array}$ & $\begin{array}{c}\text { DRV: 2,42 mm } \\
\text { (+) Abciximab } \\
\text { TCA: } 284 \text { s } \\
\text { (+) Pós-dilatação } \\
\text { Fluxo TIMI } 3 \text { pós }\end{array}$ & 363 dias & $\begin{array}{c}\text { IAM inferior } \\
\text { com elevação } \\
\text { do segmento ST }\end{array}$ & Aspirina & Definitiva \\
\hline $\begin{array}{l}\text { \# Pac, } 70 \text { anos, } \\
\text { feminino } \\
\text { Outubro } 2004 \text { - IAM } \\
\text { inferior - ICP da CD com } \\
\text { Cypher } 2,5 \times 33 \mathrm{~mm} \text { e } \\
\text { Cypher } 3,0 \times 18 \mathrm{~mm} \\
\text { FEVE: } 48 \%\end{array}$ & $\begin{array}{l}\text { DRV: 2,60 mm } \\
\text { (-) Abciximab } \\
\text { TCA: } 290 \mathrm{~s} \\
\text { (-) Pós-dilatação } \\
\text { Fluxo TIMI } 3 \text { pós }\end{array}$ & 5 dias & $\begin{array}{l}\text { IAM inferior } \\
\text { com elevação } \\
\text { do segmento } \\
\text { ST com FV }\end{array}$ & $\begin{array}{l}\text { Clopidogrel } \\
\text { Alergia a } \\
\text { aspirina }\end{array}$ & Definitiva \\
\hline $\begin{array}{l}\text { \# Pac, } 79 \text { anos, } \\
\text { masculino } \\
\text { Maio } 2005 \text { - IAM } \\
\text { anterior - ICP da DA com } \\
\text { Taxus } 3,0 \times 12 \mathrm{~mm} \text { e } \\
\text { Taxus } 2,5 \times 16 \mathrm{~mm} \\
\text { FEVE: } 32 \%\end{array}$ & $\begin{array}{l}\text { DRV: 2,46 mm } \\
\text { (+) Abciximab } \\
\text { TCA: } 252 \mathrm{~s} \\
\text { (-) Pós-dilatação } \\
\text { Fluxo TIMI } 3 \text { pós }\end{array}$ & 35 dias & $\begin{array}{c}\text { Óbito de } \\
\text { circunstâncias } \\
\text { desconhecidas }\end{array}$ & Dupla & Possível \\
\hline \multicolumn{6}{|c|}{$\begin{array}{l}(-)=\text { ausência; }(+)=\text { presença; } \mathrm{ARC}=\text { Academic Research Consortium; DRV = diâmetro de referência do vaso; FEVE = fração de } \\
\text { ejeção do ventrículo esquerdo; FV = fibrilação ventricular; IAM = infarto agudo do miocárdio; Pac = paciente; PCR = parada } \\
\text { cardiorrespiratória; TCA = tempo de coagulação ativada. } \\
\text { As linhas destacadas em cinza referem-se aos eventos relacionados aos stents convencionais e as demais, aos eventos relaciona- } \\
\text { dos aos stents farmacológicos. }\end{array}$} \\
\hline
\end{tabular}


TABELA 5

Estratificação dos eventos ao longo dos anos de seguimento

\begin{tabular}{|c|c|c|c|}
\hline & $\begin{array}{c}\text { Total } \\
n=251\end{array}$ & $\begin{array}{l}\text { Stent convencional } \\
\qquad \mathbf{n}=112\end{array}$ & $\begin{array}{l}\text { Stent farmacológico } \\
\qquad n=139\end{array}$ \\
\hline \multicolumn{4}{|l|}{ Eventos até 30 dias: } \\
\hline Óbito & 16 & 12 & 4 \\
\hline Reinfarto & 2 & 1 & 1 \\
\hline RVA & 3 & 2 & 1 \\
\hline ECAM (hierárquico) & 17 & 13 & 4 \\
\hline \multicolumn{4}{|c|}{ Eventos após 30 dias e até um ano: } \\
\hline Óbito global & 6 & 3 & 3 \\
\hline Óbito cardíaco & 4 & 2 & 2 \\
\hline Óbito não-cardíaco & 2 & 1 & 1 \\
\hline Infarto do miocárdio & 2 & 0 & 2 \\
\hline RVA & 6 & 3 & 3 \\
\hline ECAM (hierárquico) & 10 & 5 & 5 \\
\hline \multicolumn{4}{|c|}{ Eventos durante o segundo ano: } \\
\hline Óbito global & 1 & 1 & 0 \\
\hline Óbito cardíaco & 0 & 0 & 0 \\
\hline Óbito não-cardíaco & 1 & 1 & 0 \\
\hline Infarto do miocárdio & 3 & 0 & 3 \\
\hline RVA & 1 & 1 & 1 \\
\hline ECAM (hierárquico) & 5 & 1 & 4 \\
\hline \multicolumn{4}{|c|}{ Eventos durante o terceiro ano: } \\
\hline Óbito global & 2 & 2 & 0 \\
\hline Óbito cardíaco & 2 & 2 & 0 \\
\hline Óbito não-cardíaco & 0 & 0 & 0 \\
\hline Infarto do miocárdio & 3 & 2 & 1 \\
\hline RVA & 1 & 1 & 0 \\
\hline ECAM (hierárquico) & 4 & 3 & 1 \\
\hline \multicolumn{4}{|c|}{ Eventos durante o quarto ano: } \\
\hline Óbito global & 1 & 1 & 0 \\
\hline Óbito cardíaco & 1 & 1 & 0 \\
\hline Óbito não-cardíaco & 0 & 0 & 0 \\
\hline Infarto do miocárdio & 5 & 5 & 0 \\
\hline RVA & 1 & 1 & 0 \\
\hline ECAM (hierárquico) & 5 & 5 & 0 \\
\hline \multicolumn{4}{|c|}{ Eventos durante o quinto ano: } \\
\hline Óbito global & 0 & 0 & 0 \\
\hline Óbito cardíaco & 0 & 0 & 0 \\
\hline Óbito não-cardíaco & 0 & 0 & 0 \\
\hline Infarto do miocárdio & 0 & 0 & 0 \\
\hline RVA & 0 & 0 & 0 \\
\hline ECAM (hierárquico) & 0 & 0 & 0 \\
\hline
\end{tabular}

stents farmacológicos decorreu, principalmente, da diminuição da taxa de óbito cardíaco (15,9\% vs. 4,3\%; $p<0,01)$. Esse achado está de acordo com estudo recentemente publicado ${ }^{20}$. Inúmeras hipóteses, embora especulativas, podem ser aventadas para justificar esses achados, desde problemas relacionados à seleção dos pacientes, das dificuldades do ajuste nos modelos freqüentistas (que, por sua vez, afetam principalmente estudos não-randômicos), até diferenças temporais na terapia antiplaquetária. 
Magalhães MA, et al. Comparação dos Stents Farmacológicos vs. Stents Convencionais para o Tratamento do Infarto Agudo do Miocárdio com Supradesnivelamento do Segmento ST: Resultados do Registro EINSTEIN. Rev Bras Cardiol Invas. 2008;16(3):279-288.

No entanto, o grande questionamento ao implante rotineiro dos stents farmacológicos no IAMSST ainda é a questão da trombose do stent, pois, além do ambiente mais propício à ocorrência desse fenômeno, particularmente na presença de lesões complexas $^{21}$ e com alta carga trombótica ${ }^{22}$, é também difícil acessar a aderência do paciente à terapia antiplaquetária no momento da intervenção primária. A importância do clopidogrel no IAMSST é reforçada pelos dados do registro PREMIER, em que foram demonstradas elevadas taxas de mortalidade nos pacientes tratados no contexto do IAMSST com stents farmacológicos e que suspenderam o medicamento precocemente (antes de trinta dias) ${ }^{23}$. Em linha com esses achados, em nosso estudo, dos 7 casos classificados como trombose pelo critério amplo do Academic Research Consortium, apenas 2 pacientes estavam em regime antiplaquetário duplo por ocasião do evento. Esses questionamentos e achados encontram bases biológicas, como endotelização $\operatorname{tardia}^{24}$ e fenômenos mecânicos como a má aposição, inerentes ao mecanismo de ação dos stents farmacológicos de primeira geração. Particularmente, a má aposição vem sendo freqüentemente reportada no IAMSST ${ }^{19,25}$. Entretanto, o impacto clínico desses achados ainda é controverso. Nos registros RESEARCH e T-SEARCH, observaram-se fenômenos trombóticos após o primeiro e o segundo anos nos pacientes com IAMSST (4 casos) ${ }^{26}$, resultando na eliminação do benefício do stent eluidor de sirolimus em comparação com o stent convencional, no tocante à RVA. Em nossa série, apesar do número limitado de pacientes, não observamos fenômenos trombóticos muito tardios, mesmo estendendo o seguimento clínico até o quinto ano. Atribuímos esse achado, de forma especulativa, à terapia antiplaquetária dupla prolongada no grupo dos stents farmacológicos. Entretanto, devemos ressaltar que não há dados conclusivos com relação ao impacto e à duração da terapêutica antiplaquetária nesses pacientes. Recentemente, uma meta-regressão, avaliando conjuntamente mais de 4 mil pacientes em 12 estudos comparando stent farmacológico vs. convencional no infarto, não demonstrou interação entre o tempo de uso da terapia antiplaquetária e os desfechos clínicos óbito $(p=0,16)$, reinfarto $(p=0,91)$, RVA $(p=0,38)$ e trombose do stent $(p=0,26)^{27}$. Por conseguinte, ainda não está definido o tempo de antiagregação plaquetária adequado nesses pacientes. Ensaios prospectivos com seguimento clínico tardio apropriado e desenhados com esse propósito poderão fornecer as respostas clínicas necessárias para uma recomendação formal da terapêutica antiplaquetária após implante de stent farmacológico no IAMSST.

\section{Limitações}

É imperativo reconhecer todas as limitações inerentes a estudos observacionais, retrospectivos e com pequeno número de pacientes.

\section{CONCLUSÕES}

Este estudo observacional documenta o seguimento clínico tardio de pacientes pertencentes à prática clínica diária tratados por intervenção coronária primária ou de resgate no IAMSST, em que se demonstrou redução de eventos cardíacos combinados nos pacientes tratados com stents farmacológicos em comparação com os tratados com stents convencionais. No entanto, apesar de não corresponder a uma comparação randomizada "asséptica" com um grupo controle perfeitamente equilibrado, consideramos ser a informação mais relevante do estudo a ausência de eventos e de fenômenos trombóticos muito tardios (após três anos de seguimento) no grupo de pacientes tratados com stents farmacológicos. Embora atraentes, esses resultados necessitam de confirmação por outros estudos, como o HORIZONS, desenhados especificamente para responder, e formalizar de forma definitiva, às questões sobre o uso dos stents farmacológicos no IAMSST.

\section{REFERÊNCIAS BIBLIOGRÁFICAS}

1. Keeley EC, Boura JA, Grines CL. Primary angioplasty versus intravenous thrombolytic therapy for acute myocardial infarction: a quantitative review of 23 randomised trials. Lancet. 2003;361(9351):13-20.

2. Zijlstra F, Hoorntje JC, de Boer MJ, Reiffers S, Miedema K, Ottervanger JP, et al. Long-term benefit of primary angioplasty as compared with thrombolytic therapy for acute myocardial infarction. N Engl J Med. 1999;341(19):1413-9.

3. Zhu MM, Feit A, Chadow H, Alam M, Kwan T, Clark LT. Primary stent implantation compared with primary balloon angioplasty for acute myocardial infarction: a meta-analysis of randomized clinical trials. Am J Cardiol. 2001;88(3):297-301.

4. Stone GW, Grines CL, Cox DA, Garcia E, Tcheng JE, Griffin $J$, et al. Comparison of angioplasty with stenting, with or without abciximab, in acute myocardial infarction. $N$ Engl J Med. 2002;346(13):957-66.

5. Mehta RH, Harjai KJ, Cox DA, Stone GW, Brodie BR, Boura $\mathrm{J}$, et al. Comparison of coronary stenting versus conventional balloon angioplasty on five-year mortality in patients with acute myocardial infarction undergoing primary percutaneous coronary intervention. Am J Cardiol. 2005;96(7):901-6.

6. De Luca G, Suryapranata H, Stone GW, Antoniucci D, Biondi-Zoccai G, Kastrati A, et al. Coronary stenting versus balloon angioplasty for acute myocardial infarction: A metaregression analysis of randomized trials. Int J Cardiol. 2008;126(1):37-44.

7. Laarman GJ, Suttorp MJ, Dirksen MT, van Heerebeek L, Kiemeneij F, Slagboom T, et al. Paclitaxel-eluting versus uncoated stents in primary percutaneous coronary intervention. N Engl J Med. 2006;355(11):1105-13.

8. Spaulding C, Henry P, Teiger E, Beatt K, Bramucci E, Carrié D, et al. Sirolimus-eluting versus uncoated stents in acute myocardial infarction. N Engl J Med. 2006;355(11):1093-104.

9. Cutlip DE, Windecker S, Mehran R, Boam A, Cohen DJ, van Es GA, et al. Clinical end points in coronary stent trials: a case for standardized definitions. Circulation. 2007;115(17): 2344-51.

10. Reiber JH, Serruys PW, Kooijman CJ, Wijns W, Slager CJ, Gerbrands JJ, et al. Assessment of short-, medium-, and longterm variations in arterial dimensions from computer-assisted quantitation of coronary cineangiograms. Circulation. $1985 ; 71(2): 280-8$. 
11. Giglioli C, Valente S, Margheri M, Comeglio M, Chiostri M, Romano SM, et al. An angiographic evaluation of restenosis rate at a six-month follow-up of patients with ST-elevation myocardial infarction submitted to primary percutaneous coronary intervention. Int J Cardiol. 2008 (in press).

12. Steinberg DH, Pinto Slottow TL, Buch AN, Javaid A, Roy PK, Garg S, et al. Impact of in-stent restenosis on death and myocardial infarction. Am J Cardiol. 2007;100(7):1109-13.

13. Chen MS, John JM, Chew DP, Lee DS, Ellis SG, Bhatt DL. Bare metal stent restenosis is not a benign clinical entity. Am Heart J. 2006;151(6):1260-4.

14. Doyle B, Rihal CS, O'Sullivan CJ, Lennon RJ, Wiste HJ, Bell $M$, et al. Outcomes of stent thrombosis and restenosis during extended follow-up of patients treated with baremetal coronary stents. Circulation. 2007;116(21):2391-8.

15. Menichelli M, Parma A, Pucci E, Fiorilli R, De Felice F, Nazzaro $M$, et al. Randomized trial of Sirolimus-Eluting Stent Versus Bare-Metal Stent in Acute Myocardial Infarction (SESAMI). J Am Coll Cardiol. 2007;49(19):1924-30.

16. Valgimigli M, Percoco G, Malagutti P, Campo G, Ferrari F, Barbieri D, et al. Tirofiban and sirolimus-eluting stent vs abciximab and bare-metal stent for acute myocardial infarction: a randomized trial. JAMA. 2005;293(17):2109-17.

17. Valgimigli M, Campo G, Percoco G, Bolognese L, Vassanelli C, Colangelo S, et al. Comparison of angioplasty with infusion of tirofiban or abciximab and with implantation of sirolimus-eluting or uncoated stents for acute myocardial infarction: the MULTISTRATEGY randomized trial. JAMA. 2008;299(15):1788-99.

18. De Luca G, Stone GW, Suryapranata H, Laarman GJ, Menichelli M, Kaiser C, et al. Efficacy and safety of drugeluting stents in ST-segment elevation myocardial infarction: a meta-analysis of randomized trials. Int J Cardiol. 2008 (in press).

19. van der Hoeven BL, Liem SS, Jukema JW, Suraphakdee N, Putter H, Dijkstra J, et al. Sirolimus-eluting stents versus baremetal stents in patients with ST-segment elevation myocardial infarction: 9-month angiographic and intravascular ultrasound results and 12-month clinical outcome results from the MISSION! Intervention Study. J Am Coll Cardiol. 2008;51(6):618-26.

20. Hannan EL, Racz M, Walford G, Holmes DR, Jones RH, Sharma S, et al. Drug-Eluting Versus Bare-Metal Stents in the Treatment of Patients With ST-Segment Elevation Myocardial Infarction. J Am Coll Cardiol Intv. 2008;1:129-35.

21. Ong AT, Hoye A, Aoki J, van Mieghem CA, Rodriguez Granillo GA, Sonnenschein K, et al. Thirty-day incidence and six-month clinical outcome of thrombotic stent occlusion after bare-metal, sirolimus, or paclitaxel stent implantation. J Am Coll Cardiol. 2005;45(6):947-53.

22. Sianos G, Papafaklis MI, Daemen J, Vaina S, van Mieghem CA, van Domburg RT, et al. Angiographic stent thrombosis after routine use of drug-eluting stents in ST-segment elevation myocardial infarction: the importance of thrombus burden. J Am Coll Cardiol. 2007;50(7):573-83.

23. Spertus JA, Kettelkamp R, Vance C, Decker C, Jones PG, Rumsfeld JS, et al. Prevalence, predictors, and outcomes of premature discontinuation of thienopyridine therapy after drug-eluting stent placement: results from the PREMIER registry. Circulation. 2006;113(24):2803-9.

24. Virmani R, Farb A, Kolodgie FD. Histopathologic alterations after endovascular radiation and antiproliferative stents: similarities and differences. Herz. 2002;27(1):1-6.

25. Hong MK, Mintz GS, Lee CW, Park DW, Park KM, Lee BK, et al. Late stent malapposition after drug-eluting stent implantation: an intravascular ultrasound analysis with longterm follow-up. Circulation. 2006;113(3):414-9.

26. Daemen J, Tanimoto S, García-García HM, Kukreja N, van de Sande M, Sianos G, et al. Comparison of three-year clinical outcome of sirolimus- and paclitaxel-eluting stents versus bare metal stents in patients with ST-segment elevation myocardial infarction (from the RESEARCH and TSEARCH Registries). Am J Cardiol. 2007;99(8):1027-32.

27. De Luca G, Cassetti E, Marino P. Impact of duration of clopidogrel prescription on outcome of DES as compared to BMS in primary angioplasty: a meta-regression analysis of randomized trials. J Thromb Thrombolysis. 2008 (in press). 